This item was submitted to Loughborough's Research Repository by the author.

Items in Figshare are protected by copyright, with all rights reserved, unless otherwise indicated.

\title{
Variable stiffness probing systems for micro-coordinate measuring machines
}

PLEASE CITE THE PUBLISHED VERSION

http://dx.doi.org/10.1016/j.precisioneng.2015.08.004

\section{PUBLISHER}

(c) Elsevier

VERSION

AM (Accepted Manuscript)

\section{PUBLISHER STATEMENT}

This work is made available according to the conditions of the Creative Commons Attribution-NonCommercialNoDerivatives 4.0 International (CC BY-NC-ND 4.0) licence. Full details of this licence are available at: https://creativecommons.org/licenses/by-nc-nd/4.0/

\section{LICENCE}

CC BY-NC-ND 4.0

\section{REPOSITORY RECORD}

Alblalaihid, Khalid, Simon Lawes, and Peter Kinnell. 2019. "Variable Stiffness Probing Systems for Microcoordinate Measuring Machines". figshare. https://hdl.handle.net/2134/21383. 


\title{
Variable stiffness probing systems for micro-coordinate measuring machines
}

\author{
Khalid Alblalaihid $^{\mathrm{a}}$, Simon Lawes ${ }^{\mathrm{a}}$, Peter Kinnell ${ }^{\mathrm{b}, *}$ \\ a Institute for Advanced Manufacturing, The University of Nottingham, Nottingham, NG7 \\ 2RD, UK; \\ b EPSRC Centre for Innovative Manufacturing in Intelligent Automation, Wolfson School \\ of Mechanical and Manufacturing Engineering, Loughborough University, Loughborough \\ LE11 3UZ, UK
}

*Corresponding Author. Tel.: (+44) 1509227146

Email address: P.kinnell@lboro.ac.uk

\section{Abstract:}

Micro-scale probing systems are used on specialist micro-coordinate measuring machines to measure small, intricate and fragile components. Probe stiffness is a critical property of micro-scale probing systems; it influences contact force, robustness, ease of manufacture, accuracy and dynamic response. Selecting the optimum stiffness, therefore, represents a significant design challenge, and often leads to undesirable compromises. For example, when contacting fragile surfaces the probe stiffness should be low to prevent damage; however, for a more robust probing system the stiffness should be increased. This paper presents a novel concept for micro-scale probing systems with the ability to quickly and easily change and control probe stiffness during use. The intended strategy for using the proposed probe is first explained. Then the new concept is fully defined and explored through a combination of finite element analysis and experimental results. Two possible configurations of probe are described, and models for predicted performance for each are presented and compared. The models demonstrate significant stiffness reduction is possible with the proposed concept, and show it is theoretically possible to achieve a probing system with perfectly isotopic stiffness.

Keywords: Probe sensor, stiffness modulation, micro-CMM

\section{Introduction}

The continued trends of product miniaturisation, increased part complexity, and the advancing capability of high-precision manufacturing technology, has resulted in the 
development of highly accurate micro-coordinate measuring machines (micro-CMMs). At the heart of many micro-CMMs is a specialised probing system that allows tactile measurement of small, delicate and intricate parts. The probing system is a critical part of the metrology loop, and the overall accuracy of a micro-CMM is heavily dependent on the performance of the probing system [1].

To support the needs of micro-CMMs a wide range of probing systems have been reported [2-4] Conventional micro-scale probing systems typically consist of a stylus system, suspension structure and displacement transducer. A schematic representation of a conventional micro-scale probing system is shown in Figure 1, with the key elements annotated. During a measurement, when the stylus tip makes contact with a surface it is deflected from an equilibrium position. The resulting suspension structure deflection is detected using displacement transducers, and by applying the appropriate transfer function the new position of the stylus tip is calculated. While the measurement principle is similar to conventional-scale probing systems, there are a number of special features and design requirements that are specific to micro-scale probing systems.

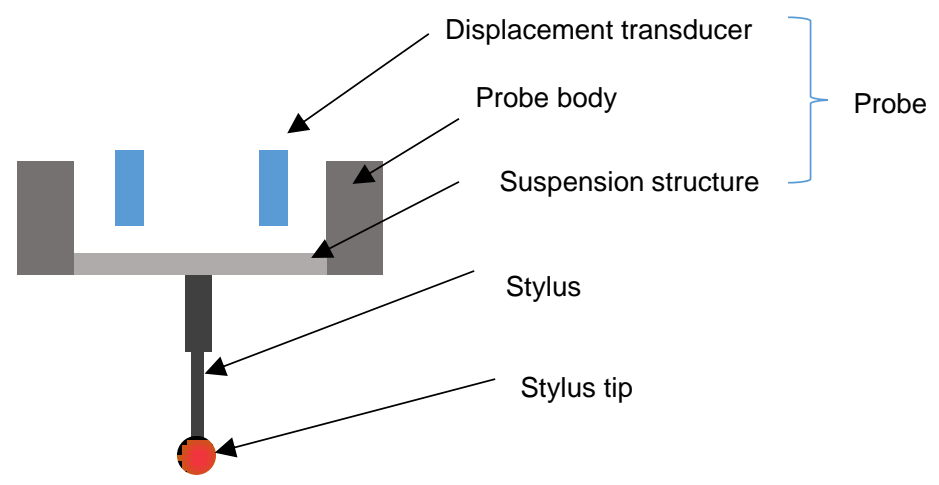

Figure 1: Schematic diagram of the probing system 
Typical micro-scale probing systems must impart low contact forces that are less than $1 \mathrm{mN}$, and ideally the contact forces should be isotropic [4]. For a given stylus tip deflection, achieving isotropic contact force requires the probe stiffness to be isotropic. Isotropic stiffness is beneficial as it reduces systematic errors during 3D measurements [5]

To allow intricate features to be measured, micro-scale probing systems are equipped with a slender stylus and small stylus tip. The stylus tip is typically around $100 \mu \mathrm{m}$ in diameter; however, smaller tips are advantageous as they allow better access to intricate features [6] . A significant problem with small stylus tips is that they can result in large contact pressure. Even with contact forces of a few millinewtons, evidence of surface damage has been observed [7]. With the trend to further reduce the stylus tip diameters, this issue becomes increasingly problematic, as contact pressure is inversely proportional to the cube root of stylus tip radius squared $[2,7]$.

To eliminate contact-induced surface damage, two options exist. The first option is to increase probe sensitivity, and reduce the probe tip velocity. This results in lower deflections of the stylus tip during a measurement, and consequently a lower contact force is developed. The second option is to decrease the probe stiffness, leading to less contact force for a given displacement [8]. In practice, reducing probe tip velocity and, therefore, increasing measurement time is rarely an attractive scenario; hence, the second option is favoured, and stiffness is reduced as much as possible.

A wide variety of low stiffness probing systems have been reported. For example, a flexure made from beryllium copper sheet, which has $50 \mu \mathrm{m}$ thickness and $15 \mathrm{~mm}$, length has been demonstrated with an isotropic stiffness of $10 \mathrm{Nm}^{-1}$ [9]. Another example is a probing system that makes use of a plate suspended by four $29 \mathrm{~mm}$ long, $180 \mu \mathrm{m}$ diameter wires. This system has anisotropic stiffness, which in the vertical and lateral direction is $11 \mathrm{Nm}^{-1}$ and 
$118 \mathrm{Nm}^{-1}$ respectively [10]. Probing systems that use silicon structures similar to commercial pressure sensors have also been developed $[11,12]$.

While low stiffness is beneficial as it reduces contact force, there are a number of negative effects that must be considered and overcome. Firstly, low stiffness probes may be sensitive to small inertial loads generated as the probe is moved by the micro-CMM. These inertial loads must not result in stylus tip displacements that are mistaken for real surface contacts. Probes that exhibit this problem require the operating speed of the micro-CMM to be reduced and, as a result, there is an unwanted increase in measurement time [8]. Secondly, as the stylus tip makes contact with a surface, relatively strong surface attraction forces occur at the contact interface $[5,8]$. These surface forces may result in the probe becoming stuck to the surface (as illustrated in Figure 2), which can lead to catastrophic failure of the probing system, and contamination of the workpiece. Thirdly, low stiffness structures are difficult to handle and manufacture, and can be easily damaged by shock loading.

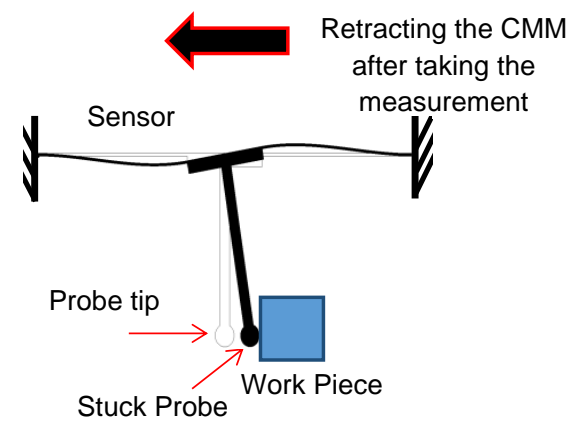

Figure 2: Large probe deflections induced as the CMM retracts the probe from a surface following a measurement.

The most promising recent work that aims to overcome some of these problems has resulted in the development of a novel vibrating micro-scale probing system [8]. This probing system has been designed to counteract surface attraction force by using the dynamic inertia of the vibrating stylus system. However, the operation of this probing system requires a 
complex control strategy, and the exact nature of the interaction between the vibrating stylus tip and the surface has yet to be fully described.

In this paper, a completely new concept for probing systems with tuneable stiffness is presented. The new concept is fully defined and a description of the envisaged operating procedure for the probing system is presented. The proposed probing system uses a suspension structure that can be tuned to have either low or high stiffness as required. To illustrate this concept two examples of this kind of suspension structure are presented for comparison. The capabilities of each structure are carefully considered by using finite element analysis to model their potential performance.

To further demonstrate the feasibility of the proposed concept, a prototype probing system has been produced and results from initial testing are presented. The full capability of the variable stiffness probe is demonstrated by using a combination of the experimental data and finite element modelling, and the influence of critical geometric features that affect the performance are presented. The possible sources of error resulting from the approach have also been taken into account, and for each of the described potential issues, control methods are suggested to mitigate these errors.

\section{Variable stiffness probing systems}

Within a micro-scale probing system, the suspension structure (see Figure 1) has the main effect on probe stiffness. To modify the stiffness of the suspension structure during use, three strategies can be adopted. The first strategy is to apply additional force to the structure to augment the action of external loads applied to the stylus tip $[13,14]$. The second strategy is to modify the effective geometry of the structure; for example, by moving the fixing points of the suspension structure to create either a shorter or longer beam [15]. The third strategy is to 
subject the suspension structure to an internal strain. This results in either strain stiffening for tensile loads, or reduced stiffness for compressive loads [16]. In previous work, the potential of each of these strategies was compared [17], and it was concluded that the use of compressive strain to reduce stiffness is the most suitable method for use with micro-scale probing systems.

To create a variable stiffness probing system, a novel suspension structure was designed to allow compressive force to be applied to the main spring elements within the structure. In general, as long as the spring elements are made of long slender beams onto which a compressive load can be applied, then a wide range of geometries is possible. Loads can be applied to the ends of each beam using a piezo-electric or other similar form of actuator. Using this type of structure, a probing system can be designed to operate in either a "stiff"or "flexible" mode. These modes may then be selected at suitable points within a measurement cycle by energising the actuator, as illustrated by Figure 3. For example, when the stylus tip is being driven towards a workpiece, the probe will be in the stiff mode (Figure 3a) to prevent false triggers caused by inertial loads generated when the probing system is moved by the micro-CMM. Immediately prior to making contact, when the probe enters a predetermined distance from the expected surface, it is set to the low stiffness condition (Figure 3b) to ensure minimal contact force. After a measurement has been made, when the probe must be removed from the surface, it can be switched back to the stiff mode (see Figure 3c). In this mode, it is possible to develop sufficient force at the stylus tip to overcome surface attraction forces, without overstraining, and potentially damaging, the suspension structure. 

a) Stylus tip
b) Stylus tip makes
c) Retracting the stylus
approaching work piece
contact with the work
tip after contact
piece

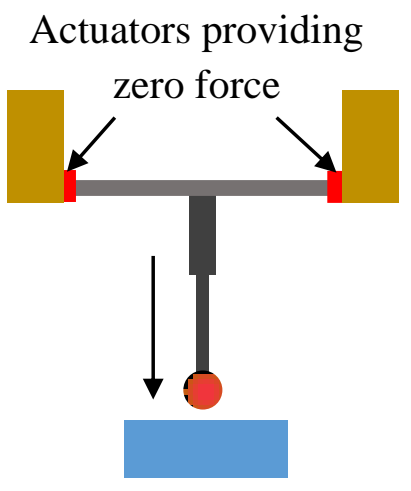

Actuators providing

Actuators providing

compressive force
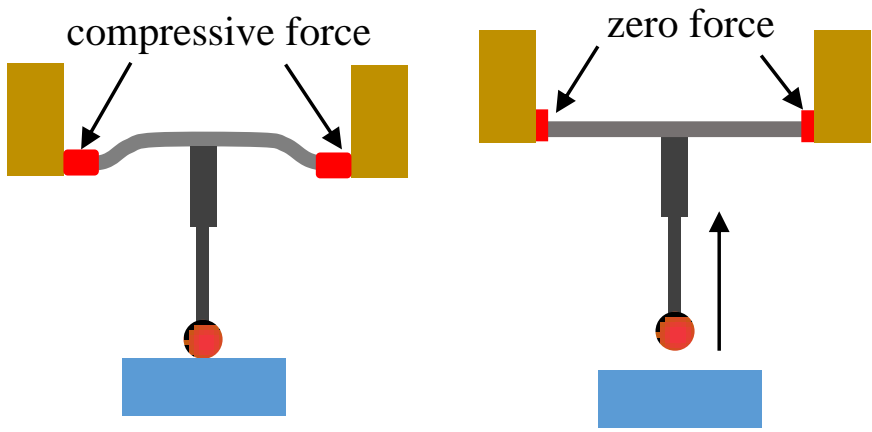

Figure 3: Illustration of the application of a variable stiffness probing system. (a) The suspension structure is in a stiff mode where the stylus tip is approaching the target surface; (b) in the flexible mode while the micro-CMM is making the measurements; (c) in the stiff mode when the measurement has been taken and the stylus tip is retracted.

When designing the suspension structure, the aim is to allow the ends of the long slender beams to be constrained in a clamped and guided configuration, so that external compressive loads can easily be applied using an external actuator. Figure 4 shows two examples of suspension structure configurations, the clamped and guided beam constraints are represented by guided sets of rollers. For each suspension structure, an intermediate body links the beams and provides a mounting point for the stylus. To reduce the stiffness of each structure, a set of compressive forces should be applied at the positions indicated by the arrows in Figure 4. The compressive forces cause the stiffness of the beams to reduce until they become zero at the buckling point.

\section{Suspension structure performance considerations}


Suspension structures with a range of geometric configurations are potentially possible as illustrated by the two very different examples shown in Figure 4. Figure 4(a) illustrates the tri-beam design which allows axial force to be applied through each beam with an intersect point at the centre of the intermediate body; Figure 4(b) shows the delta-beam design which consists of three segments, each with two beams. In the delta-beam design, axial load is applied to the ends of each beam, with equal magnitude and opposite direction. Both configurations are also designed such that, if equal loads are applied, there is no resulting displacement of the central intermediate body.

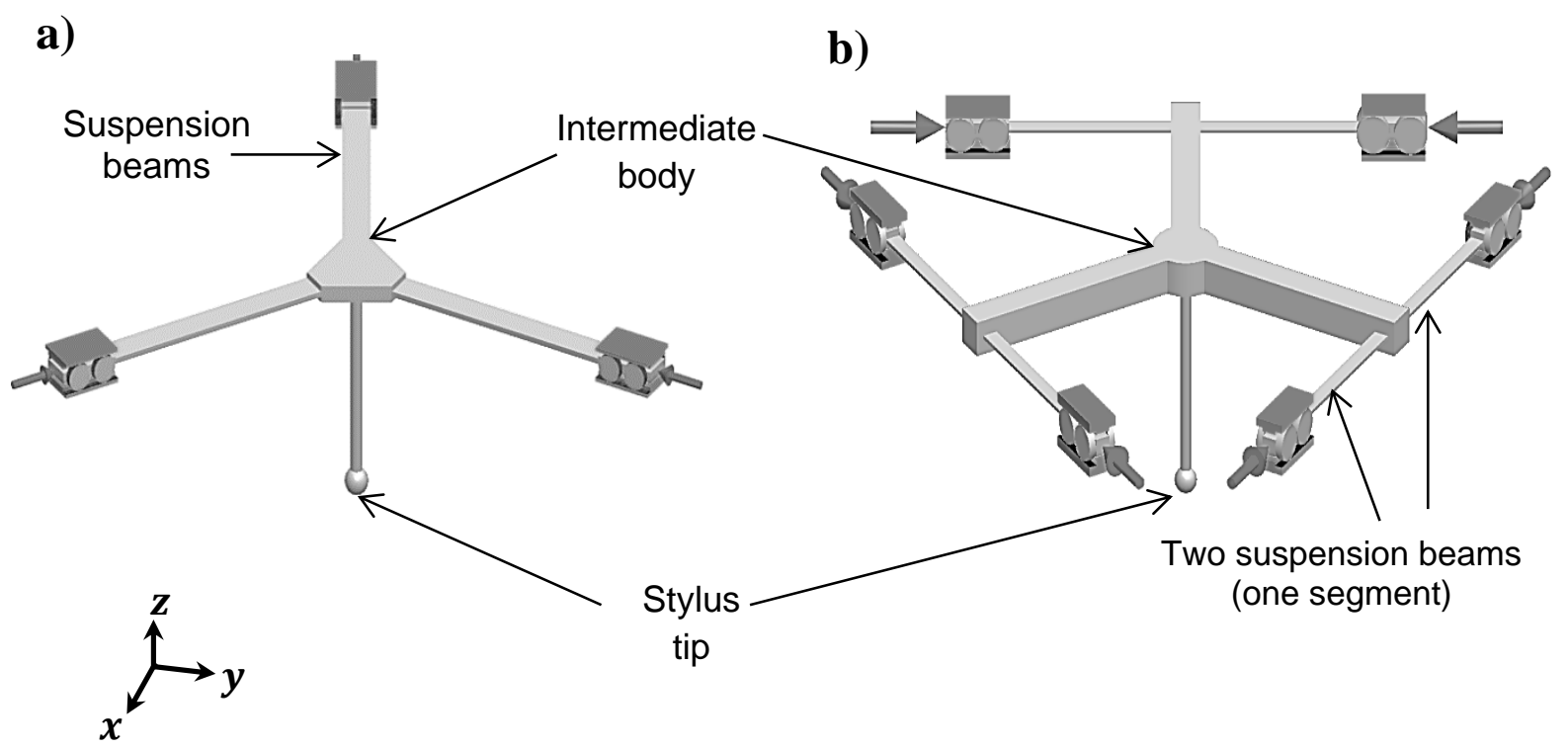

Figure 4: (a) Tri-beam design which consists of three suspension beams, joined to the intermediate body, (b) delta-beam design consisting of six suspension beams connected to a larger intermediate body. Guided roller constrains are shown at the ends of each of the beams for illustration only to indicate a degree of freedom allowing axial load to be applied, with the arrows used to indicate applied load vectors.

Qualitatively, the two structures have different strengths and weaknesses. The tri-beam structure has the advantage of being constrained in only three positions, and only three actuators are needed to load the structure, whereas six are needed for the delta-beam 
structure. An additional benefit of the tri-beam structure, as compared to the delta-beam structure, is that the three beams are spaced to allow sufficient room for actuators to be integrated. The disadvantage of the tri-beam structure is that an equal load must be applied to each beam to avoid translation of the intermediate body. This is not the case for the deltabeam design which only requires equal loads to be applied to each individual beam.

The ability to vary the loads applied to each beam may be beneficial as it would allow independent stiffness control of each beam. For example, independent stiffness control would be useful to compensate for errors in manufacturing that resulted in beams with different geometry. In this case, different loads could be independently applied to each beam; which would allow each beam to be tuned to a uniform stiffness. A negative consequence of the delta-beam structure, as compared to the tri-beam structure, is that it requires a larger intermediate body which increases probe inertia.

To provide a more detailed understanding of how the two presented structures behave, finite element models of both types were created using COMSOL 4.3a finite element software. Using the finite element models, the vertical and lateral stiffness of each suspension structure was estimated. For each structure, either a force or a moment load was applied to the centre of the intermediate body, and the finite element models were run to determine probe tip displacement. A direct force of $1 \mathrm{mN}$ was applied to assess stiffness in the Zdirection (as defined in Figure 4). To assess stiffness in the $\mathrm{X}$ and $\mathrm{Y}$ directions, a moment load was applied. The applied moment was selected to be representative of a stylus tip load of $1 \mathrm{mN}$, which was chosen as it represents an upper limit of the range of achieved probing forces for many micro-scale probing systems [3]. According to Pril [18], a stylus length of $4 \mathrm{~mm}$ should provide good accessibility and visibility for typical micro-CMM applications. Thus, to allow a range of stylus lengths to be considered, three moment loads of $3.8 \mu \mathrm{Nm}$, $6.6 \mu \mathrm{Nm}$ and $8 \mu \mathrm{Nm}$, were applied at the centre of the intermediate body. These moments 
represent the force of $1 \mathrm{mN}$ acting at the end of a $3.8 \mathrm{~mm}, 6.6 \mathrm{~mm}$ and $8 \mathrm{~mm}$ long stylus respectively. The geometric parameters that define each of the structures are presented in Figure 5 and detailed in Table 1. The material properties used are those of titanium, Grade 5 (see Table 2). Titanium was chosen as it has a high strength to weight ratio which assists to reduce the effective mass of the suspension structure. Titanium also has a low elastic modulus and high yield strength, which is beneficial to reduce stiffness and minimise hysteresis.

a)

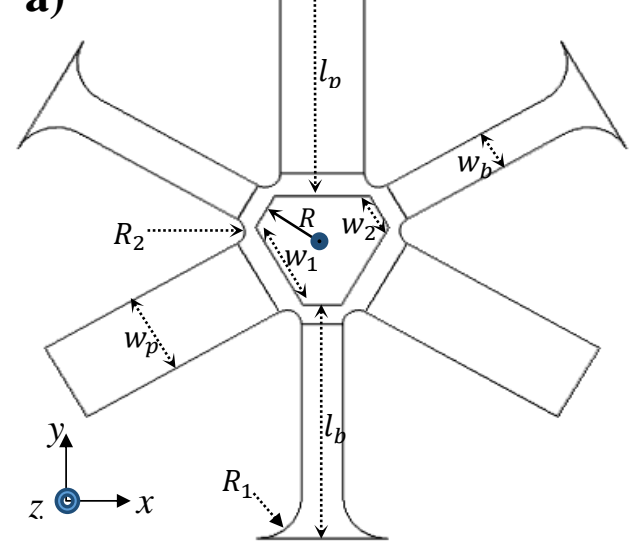

b)

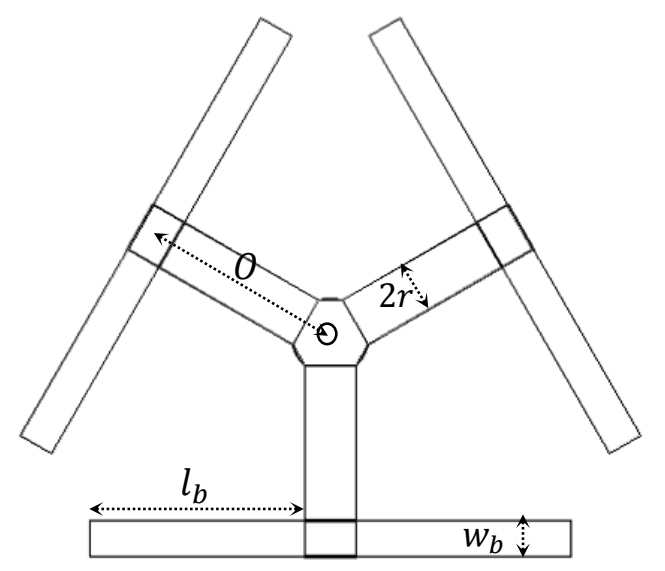

Figure 5: A main geometric parameters of the two considered design variants. (a) The tribeam design and (b) The delta-beam design.

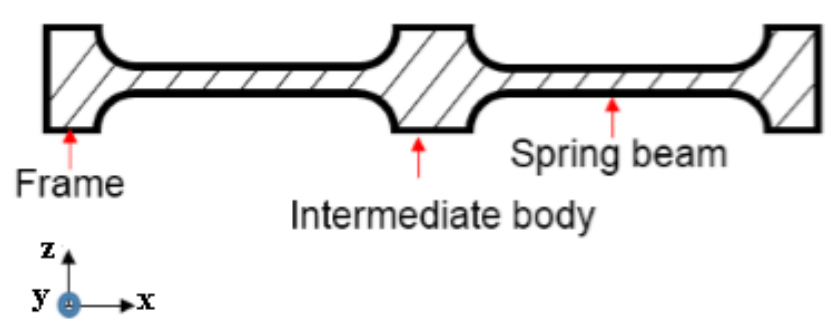

Figure 6: Schematic diagram of the cross-section of a beam to demonstrate the symmetry along the $z$-direction.

Table 1: Structure main parameters of the tri-beam and the delta-beam design (define all terms) 


\begin{tabular}{|c|c|c|}
\hline $\begin{array}{l}\text { Elastic } \\
\text { spring }\end{array}$ & $\begin{array}{l}l_{b}=4215 \mu \mathrm{m}, R_{1}=720 \mu \mathrm{m}, \\
w_{b}=630 \mu \mathrm{m}, \\
t_{b}=110 \mu \mathrm{m} \text { (thickness) }\end{array}$ & $\begin{array}{l}l_{b}=4215 \mu \mathrm{m}, \\
w_{b}=630 \mu \mathrm{m}, \\
t_{b}=110 \mu \mathrm{m} \text { (thickness), }\end{array}$ \\
\hline $\begin{array}{l}\text { Sensor } \\
\text { paddles }\end{array}$ & $\begin{array}{l}l_{p}=4184.6 \mu \mathrm{m}, R_{2}=450 \mu \mathrm{m} \\
w_{p}=1300 \mu \mathrm{m}\end{array}$ & - \\
\hline $\begin{array}{l}\text { Intermediate } \\
\text { body }\end{array}$ & $\begin{array}{l}R=1085 \mu \mathrm{m}, \\
w_{1}=1574.2 \mu \mathrm{m}, w_{1}=610 \mu \mathrm{m}\end{array}$ & $\begin{aligned} O & =3926 \mu \mathrm{m} \\
r & =500 \mu \mathrm{m}\end{aligned}$ \\
\hline
\end{tabular}

Table 2: Material properties of the two configuration designs (titanium, Grade 5) [19]

\begin{tabular}{llll}
\hline $\begin{array}{l}\text { Elastic modulus, } \\
\mathrm{E}(\mathrm{GPa})\end{array}$ & $\begin{array}{l}\text { Poisson's } \\
\text { ratio, } \nu\end{array}$ & $\begin{array}{l}\text { Density, } \rho \\
\left(\mathrm{kg} \mathrm{m}^{-3}\right)\end{array}$ & $\begin{array}{l}\text { Yield strength, } \\
(\mathrm{MPa})\end{array}$ \\
\hline 113.8 & 0.342 & 4430 & 880 \\
\hline
\end{tabular}

Using the finite element models, the vertical $(k z)$ and lateral $(k y)$ stiffness for each design was evaluated for a range of axial loads applied to the suspension structure beams. In the model, these loads were applied in the form of forced axial displacements of the beam loading points. The results from this analysis are illustrated in Figure 7, which shows the relationship between applied axial displacement and stiffness, for three stylus lengths.

The effect of load on lateral and vertical stiffness for both structures is not equal. From Figure 7 this is clearly observed by the change in slope of the curves for vertical and lateral stiffness. An important consequence of this is that an intersection point of the curves must exist, and at this point the probe stiffness is isotropic. In Figure 7, the isotropic stiffness point with a $3.8 \mathrm{~mm}$ stylus length occurred at $1.14 \mathrm{~N} \mathrm{~mm}^{-1}$ and $5 \mathrm{~N} \mathrm{~mm}^{-1}$ for the tri-beams and delta-beam design respectively. This is an important observation, as achieving isotropic stiffness by geometric design alone is not easy and in some cases not possible. Therefore, a major benefit for probe sensors with tuneable stiffness is the ability to operate at the isotropic stiffness point. It is also evident from Figure 7 that changing the stylus length leads to a shift in the position of the isotropic stiffness point. Therefore, for any future application of variable 
stiffness suspension structures, consideration would be required to ensure the correct isotropic stiffness point was achieved for each stylus length required.

a)

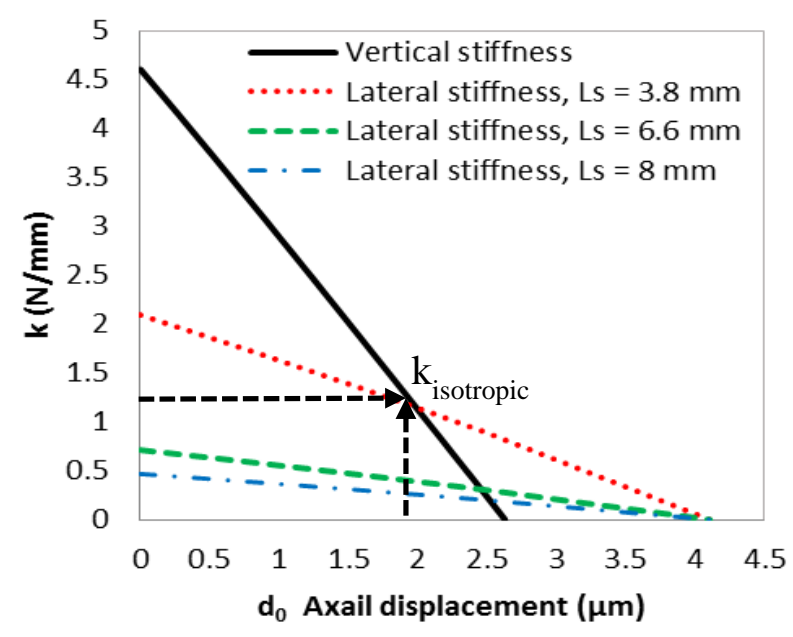

b)

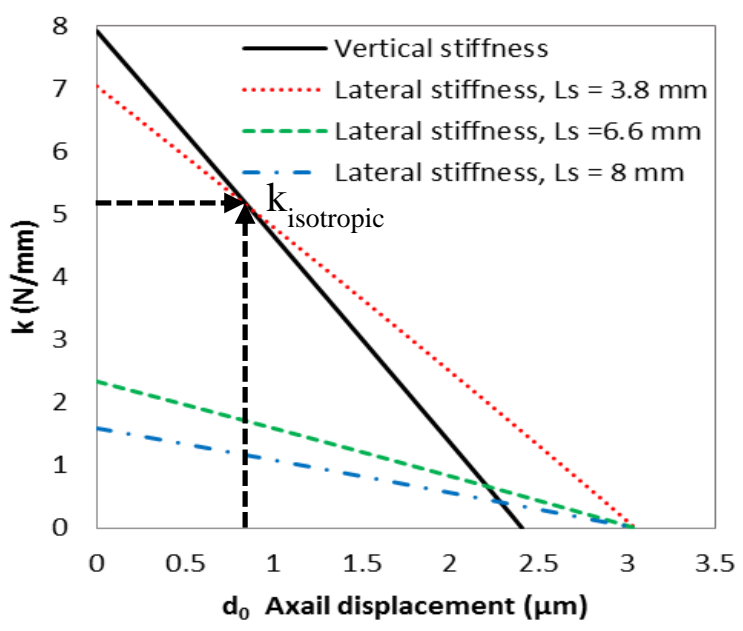

Figure 7: Stiffness versus axial displacement determined by FEA for (a) the tri-beam and (b) delta-beam design. Different effective stylus lengths ( $l \mathrm{~s}=3.8 \mathrm{~mm}, 6.6 \mathrm{~mm}$ and $8 \mathrm{~mm}$ ) have been considered.

An additional consideration is that it is advantageous to have curves for both the vertical and lateral stiffness that are closely matched in slope. This means that, for a given difference in vertical and lateral stiffness, the range of suitable applied loads is increased. To illustrate this Figure 8 shows the ratio of the vertical to the lateral stiffness as a function of applied load. From Figure 8 , it is clear that the delta-beam design (3.8 $\mathrm{mm}$ stylus length) remains within a stiffness mismatch of $10 \%$ or less for a larger range of applied displacements as compared to the tri-beam design. 


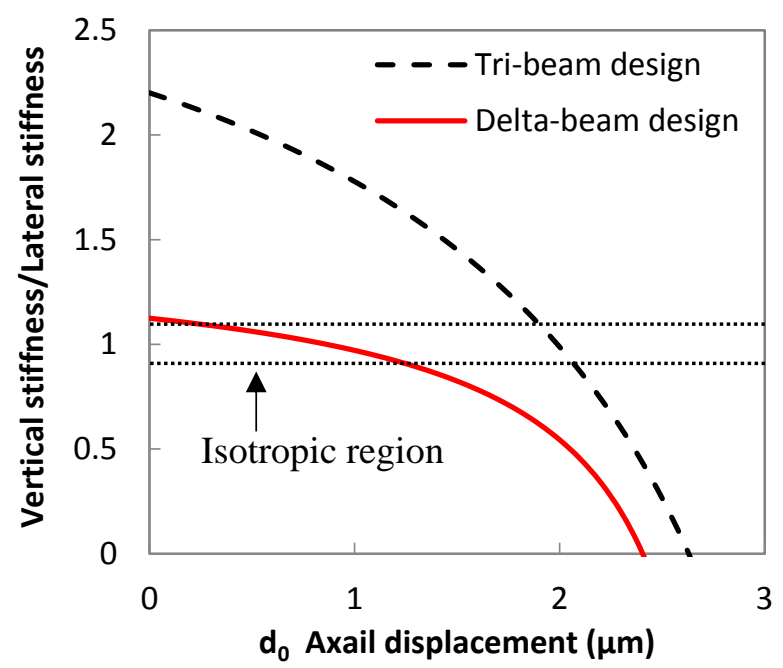

Figure 8: A plot of the ratio of the vertical to lateral stiffness as a function of the axial displacement $\left(d_{o}\right)$ for both delta- and tri-beam designs, with $3.8 \mathrm{~mm}$ stylus length.

\section{Probe performance analysis}

To provide an example of a full probing system, a tri-beam variable stiffness suspension structure was integrated with a stylus system and displacement sensors. The suspension structure was manufactured using chemical etching, as this technique provides high process control and allows precision parts to be manufactured in batches, making it a cost effective technology. However, due to limitations with the chemical etching process that restricted the thickness of the components, the suspension structure had to be manufactured in three separate layers [20]. The middle layers consist of the spring beams, sensor paddles and intermediate body. The top and bottom layers were produced to sandwich the middle layer and are both identical, and only contain the features associated with the outer frame, as can be seen in Figure 9(a). The main features of the outer frame with the suspension structure in place can be seen in Figure 9(b). Compliant mechanisms were included as a means of providing a clamped-guided constraint to the spring beams. The compliant mechanisms do this by providing good rotational constraint, but still allow lateral movement so axial compression of the beams is possible. This is achieved by linking two spatially separated parallel beams to form an 'H-shaped' structure. 
To allow measurements of the suspension structure deflections during probing, the intermediate body has three cantilevered sensor paddles attached to it. The sensor paddles are used to provide a target surface for three capacitive displacement sensors that are aligned above the sensor paddles at a stand-off distance of approximately $100 \mu \mathrm{m}$.

a)

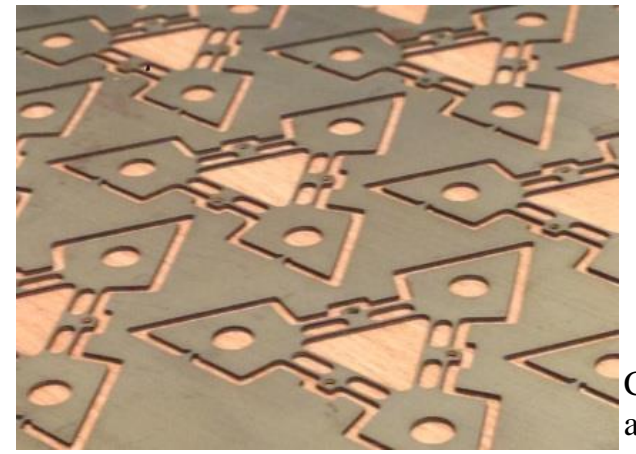

b)

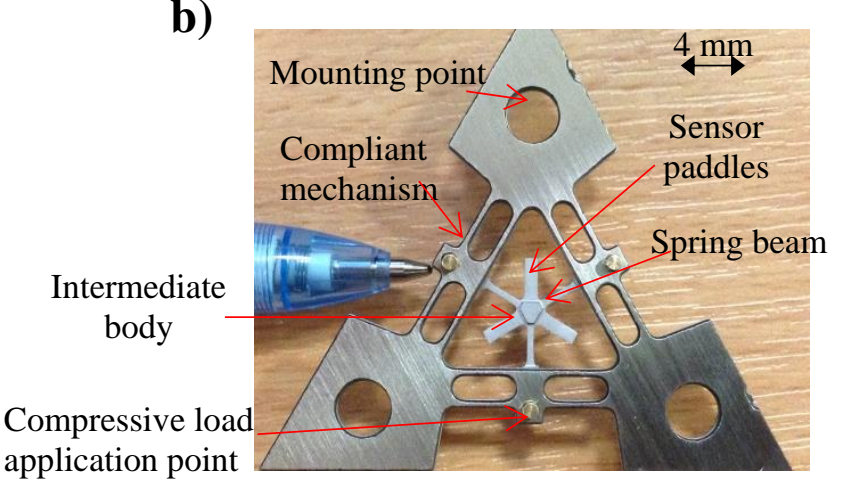

Figure 9: a) The spring steel sheet has been etched to produce the top and bottom layers. b) The prototype of the smart suspension structure, with main structural elements labelled where the middle layer (suspension structure) is made of titanium.

The geometry of the manufactured suspension structure is as described in Table 1. To provide a reasonable representation of a full probing system, a modified commercial stylus system (Renishaw stylus, serial number A-5000-7800) was bonded using a fast acting cyanoacrylate adhesive to the intermediate body. This stylus has total length of $6.6 \mathrm{~mm}$ and conical neck which holds the stem. The modified geometry of the stylus is illustrated in Figure 10, and the dimensional parameters and material properties are defined in Table 3 and Table 4 respectively. 
a) Upper and lower plate

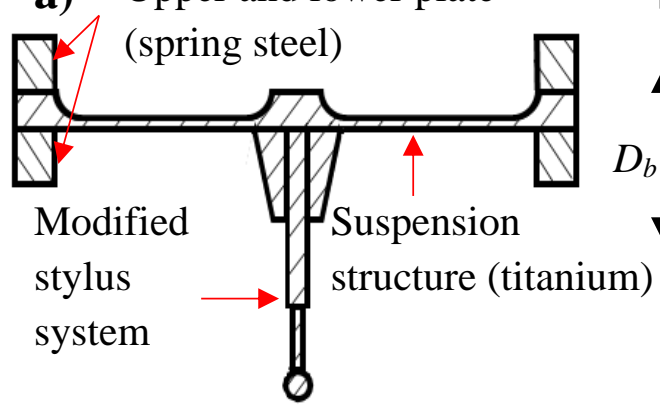

b)

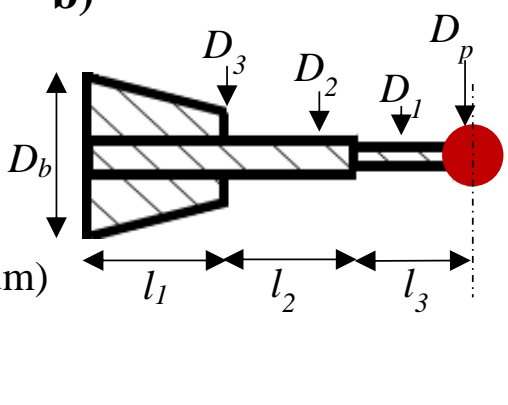

Figure 10: (a) Schematic cross-sectional diagram of the probing system (b) The main geometric parameters of modified stylus system.

Table 3: A main parameters of the modified micro-probe

\begin{tabular}{ll}
\hline & Modified micro-probe \\
\hline Item & Specifications \\
\hline Ruby ball & $D_{p}=300 \mu \mathrm{m}$, \\
Stem & $D_{1}=210 \mu \mathrm{m}, D_{2}=675 \mu \mathrm{m}, l_{2}=l_{3}=2000 \mu \mathrm{m}$ \\
Stem holder & $D_{3}=1314.2 \mu \mathrm{m}, D_{b}=2560 \mu \mathrm{m}, l_{1}=2600 \mu \mathrm{m}$ \\
\hline
\end{tabular}

Table 4: Material properties of the micro-probe (Renishaw, A-5000-7800) [21]

\begin{tabular}{llll}
\hline Material & $\begin{array}{l}\text { Elastic modulus, E } \\
(\mathrm{GPa})\end{array}$ & $\begin{array}{l}\text { Poisson's } \\
\text { ratio, } v\end{array}$ & $\begin{array}{l}\text { Density, } \rho \\
\left(\mathrm{kg} \mathrm{m}^{-3}\right)\end{array}$ \\
\hline Ruby ball & 375 & 0.22 & 3890 \\
\hline Tungsten carbide & 345 & 0.31 & 19300 \\
\hline
\end{tabular}

The assembled probing system was mounted on a fixture containing three piezoelectric actuators, and the actuators were aligned to the load application points on each compliant mechanism. A photograph of the probing system with actuators (piezoelectric stacks, PICMA, P-882.11) attached is shown in Figure 11. 


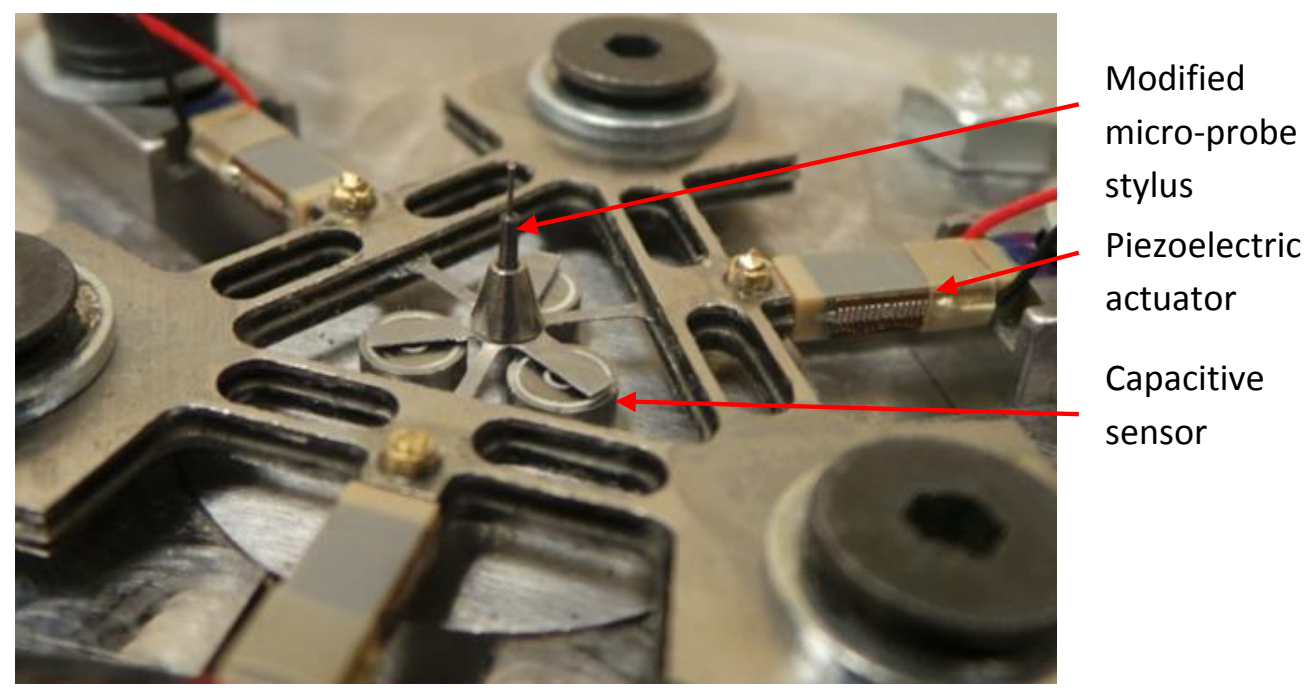

Figure 11: Assembled probing system, with three mounted piezoelectric actuators for compressive force application, and beneath the suspension structure three capacitive sensors.

To test the ability of the actuators to reduce the stiffness of the suspension structure, a range of loads were applied using the actuators, and the resulting modal frequencies of the structure were monitored using the three capacitive sensors. This provided a simple and effective method for monitoring structural stiffness as a function of applied load, as both the vertical and lateral stiffness can be easily related to specific modal frequencies of the structure. To identify the expected vibration modes of the probing system, modes representative of vertical and lateral stiffness were estimated using finite element analysis of the full structure including the stylus system, and the selected mode shapes can be seen in Figure 12. Under zero applied load the vertical and torsional frequencies were estimated to be $1114 \mathrm{~Hz}$ and $1423 \mathrm{~Hz}$ respectively. 
a)

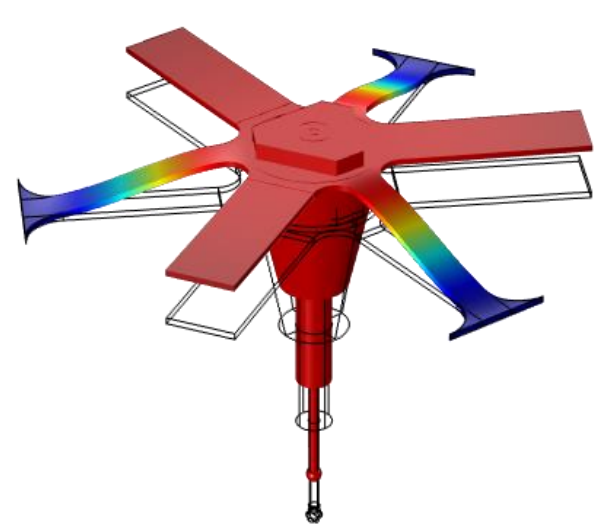

b)

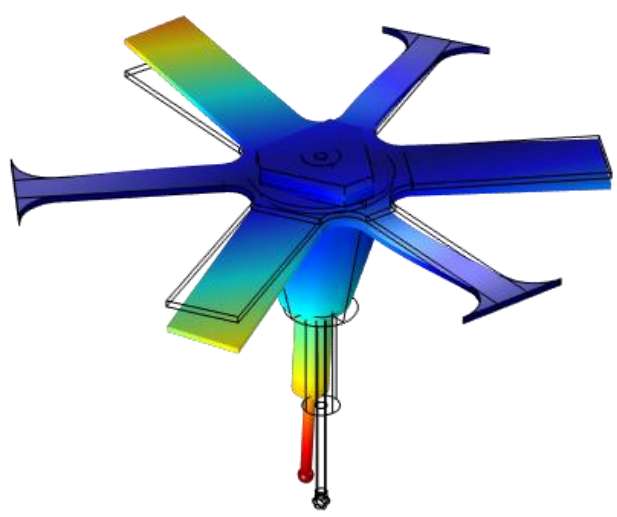

Figure 12: Shows mode shapes for zero applied compressive load: (a.) the first mode ( $f=1114 \mathrm{~Hz}$ ), where the full micro-probe vibrates vertically, and (b.) the second mode $(f=1423 \mathrm{~Hz})$ where the centre of the micro-probe has a rotational motion.

The model was then used to predict how the vertical and torsional frequencies would change as compressive load was applied to the spring beams. To experimentally validate the finite element model, the vertical and torsional frequencies were also measured using the prototype device. To make this possible, the actuators were used to apply a combined static and dynamic load. The static load was a voltage that was sequentially increased from zero to $90 \mathrm{~V}$ to apply an increasing compressive load to the beams. The dynamic component was a sinusoidal chirp signal of $0.5 \mathrm{~V}$ peak-to-peak. This chirp signal was used to excite the structure through a range of frequencies from $100 \mathrm{~Hz}$ to $2000 \mathrm{~Hz}$. The capacitive sensors, that are located below the sensor paddles as illustrated in Figure 11, were used to detect the resulting resonant frequencies. Both the experimental data, and the data generated from the finite element model are shown in Figure 13. A direct comparison is not possible as the transfer function of the actuators was not determined; however, both sets of data present the same underlying characteristics. Most interestingly, it is clear that the vertical and torsional frequency reduction is limited by a turning point, beyond which the structural frequencies increase. 
The cause of this turning point was determined to be a combination of factors that result in a non-symmetrical strain state being created within each suspension beam. This causes the suspension structure to be subjected to a small degree of bending, as opposed to the intended completely symmetrical plain compressive strain. These bending stresses predispose the system to bend before it buckles, and when bending occurs, there is a relief in the applied compressive load. This is a non-linear effect, and as such it is not dominant until a significant level of compressive load has been applied. The non-linear nature results in the turning point observed from both the experimental and numerical performance data.

a)

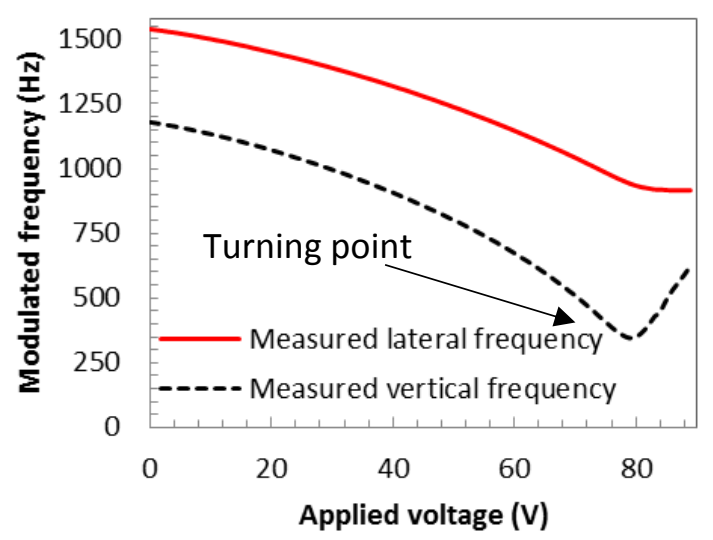

b)

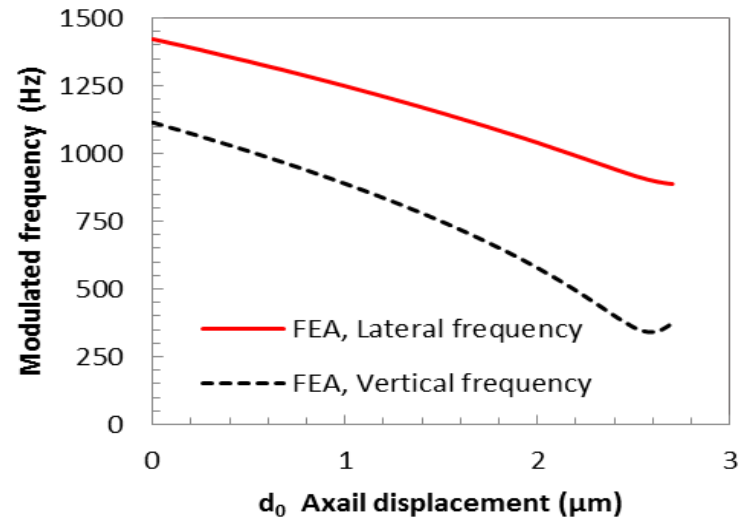

Figure 13: (a) A plot of the vertical and the torsional frequency of the full micro-probe against the applied static voltage determined experimentally for $6.6 \mathrm{~mm}$ stylus length. (b) The modulated frequencies of the full probe as estimated by FEA analysis plotted as function of axial displacement $\left(d_{o}\right)$. 
Two potential causes of the non-symmetrical strain state were considered. The first was the non-symmetrical geometry that results from the chemical etching process, and the addition of the stylus system to the suspension structure, as illustrated in Figure 10(a). Note that the intermediate body when coupled to the stylus system presents an asymmetric stiffness. The stylus base is larger and provides more reinforcement when compared to the intermediate body itself. This result in small rotation at the ends of the spring beams in reaction to the applied compressive load, causing the beams to bend. The second cause, was the influence of gravity on the structure providing additional non-symmetrical load, especially as the stylus system is relatively massive compared to the suspension beams. To explore the impact of these factors, the finite element simulation was adjusted to demonstrate the sensitivity of the structure to variations in both the mass of the stylus as well as structural asymmetry. Considering gravitational loading first, the simulation was adjusted to zero gravity load. The results for zero gravity are compared to the previous results in Figure 14, and it can be seen that gravity has a negligible effect on the device. This leaves structural asymmetry as the most likely cause of the turning point.

To compensate for the asymmetrical geometry, the finite element model was adjusted by trial and error until a perfectly balanced load was achieved in the suspension beams. In this context a balanced load in the suspension beams is one where the forces exerted by the beams on the intermediate body result in no motion of the intermediate body. The end points of the beams therefore remain stationary and as the system approaches buckling it can be considered to be fixed-fixed. It was found that this can be achieved by changing parameter $D b$ (as show in Figure 10), and that a value of $2146 \mu \mathrm{m}$ resulted in vertical and lateral frequencies that could be reduced down to zero, as shown in Figure 14. It must be noted that the effective mass of the probe was reduced $(72 \mathrm{mg})$ as compared to the initial model (104 $\mathrm{mg}$ ), and this reduction in mass and the stiffness (see Figure 15) leads to slightly increased 
initial frequencies. Nevertheless, the finite element model clearly indicates that, through careful mechanical design, it is possible to design a structure capable of generating plain compressive stress in the suspension beams, and that this structure does not have to be geometrically symmetrical.

In addition to the modal frequency analysis described above, the finite element model was also used to predict the stiffness in the vertical and lateral directions of the suspension structure as a function of axial displacement. Figure 15 shows the predicated stiffness as a function of axial displacement for two cases. The first case illustrates the reduction of the stiffness for the manufactured geometry, with parameters of the modified robe in Table 3. The lowest stiffness that can be achieved in the vertical and lateral direction at the turning point is around $0.44 \mathrm{~N} \mathrm{~mm}^{-1}$ and $0.34 \mathrm{~N} \mathrm{~mm}^{-1}$ respectively. The initial ratio between the vertical and lateral stiffness is reduced from 7 to 1.3 , which is close to the isotropic stiffness. However, to achieve the fully isotropic stiffness, the structure with optimised geometry for a balanced load must be used; as can be seen in Figure 15 this results in a crossing point for the lateral and vertical stiffness curves. 


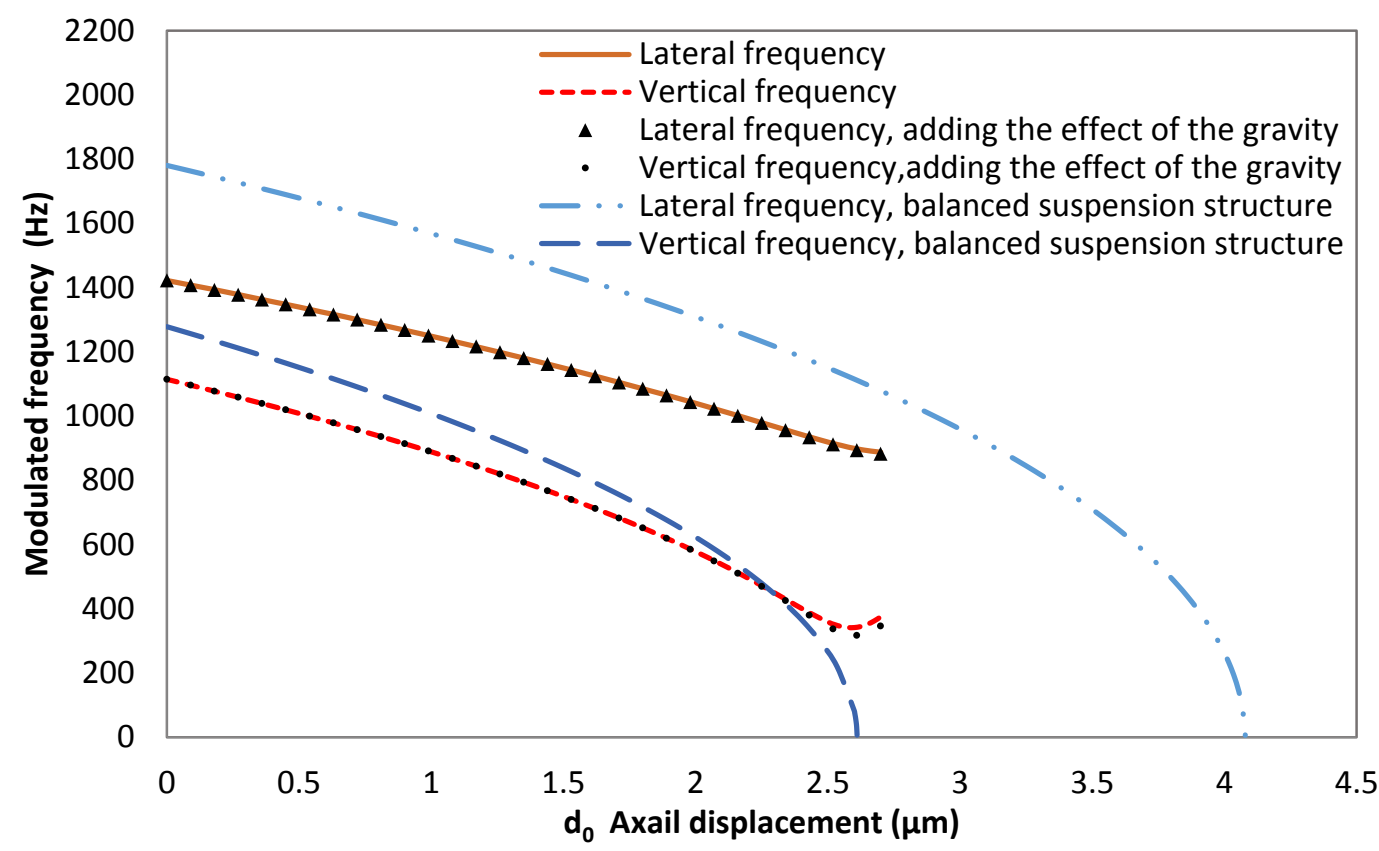

Figure 14: A modulated frequencies of the full micro-probe, estimated by FEA and plotted as a function of axial displacement $\left(d_{o}\right)$. Three conditions are considered: manufactured geometry, manufactured geometry with gravity and a structure with optimised geometry to support a balanced load.

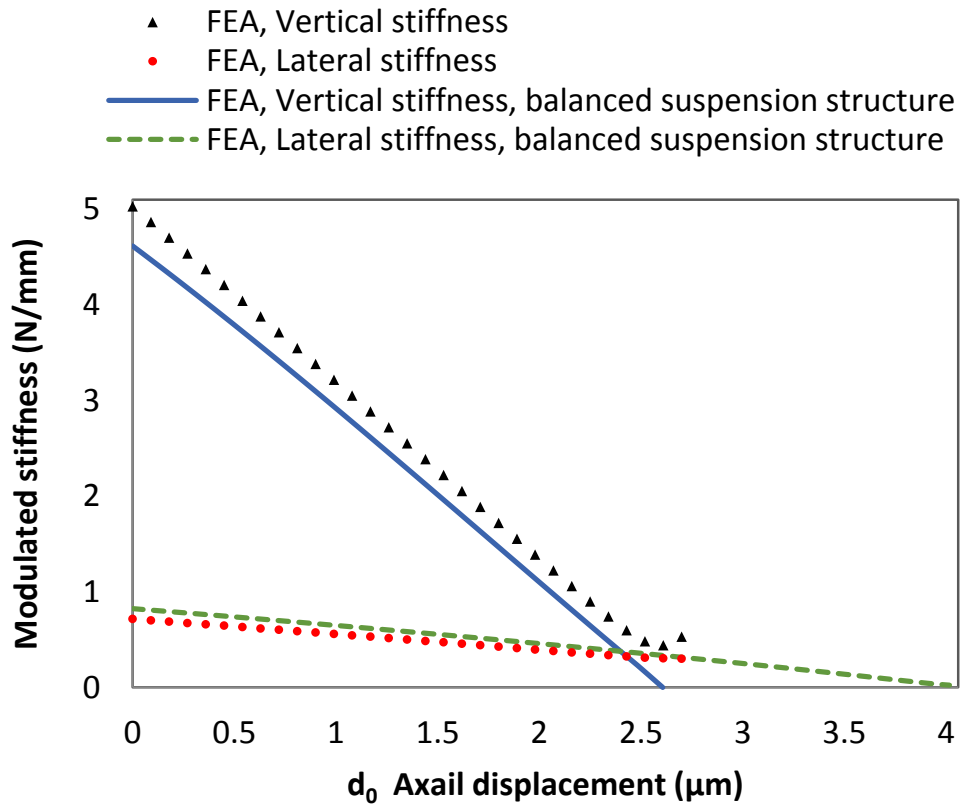

Figure 15: A modulated stiffness of the full micro-probe, estimated by FEA and plotted as a function of axial displacement $\left(d_{o}\right)$. Two conditions are considered: manufactured geometry and a structure with optimised geometry to support a balanced load. 


\section{Discussion of potential error sources}

The variable stiffness micro-scale probing system represents an extension of current and proven probe technology. However, to find application in metrology it is important to understand if this capability introduces any new sources of error. The main concern when using this technique is that as compressive load is applied to the beams, therefore, they suffer some degree of bending which results in a deflection of the probe tip. It has been shown that if asymmetry is introduced in to the system, then through careful design it is possible to reduce these bending loads to zero; however, in reality it is expected that there will always be imperfections in the structure that will not be compensated. As a result, probe tip deflection resulting from the applied compressive loads must be expected. This may be acceptable providing the load can be applied in a repeatable way. To ensure the applied load is repeatable, it is proposed that the three capacitive sensors are used to monitor the deflection of the suspension structure caused by the application of compressive loads.

An error that is more difficult to deal with would arise if the applied axial force is not applied equally to all three beams. This would result in a lateral in-plane displacement of the central intermediate body. The three capacitive sensors would not be able to detect this type of error as they are only sensitive to vertical displacements. In this case, it is proposed that the sensors may be used to monitor the dynamic response of the probe, as the modal frequency response of the structure would be modified as a result of uneven applied loads.

\section{Outlook and future work}

An innovative solution for creating a novel variable stiffness micro-scale probing system has been presented, and the initial proof-of-principle for this concept has been provided, with experimental data from a prototype probing system. This variable stiffness probing system 
demonstrates promising behaviour that may well be able to overcome some significant challenges related to the manufacture and application of micro-scale tactile probing systems.

It has been shown that using this proposed method, a suspension structure may be designed to operate in two distinct modes. The first mode should allow the probing system to overcome surface adhesion forces without damage. This mode also results in a sufficiently stiff probe that can eliminate inertial false triggering, which allows for a faster operating speed for the micro-CMM. The second mode results in a flexible probe which allows a suitably low contact force between the measurement surface and the stylus tip; this is important for contacting fragile components without damage. It has also been shown that, by proper design, the probe is able to operate in a fully isotropic stiffness regime.

Of the two suspension structures presented, the geometric simplicity of the tri-beam design offers a lower stiffness and easier manufacturing. However, with the tri-beam structure there is a requirement to apply equal forces to each of the beams in order to maintain probe position. The delta-beam design by comparison permits compressive forces to vary between beam elements. The delta-beam also demonstrates more closely matched vertical and lateral stiffness, thereby extending the isotropic stiffness regime. The two very different embodiments of the suspension structure highlight the flexibility of the concept, and illustrates that further work is required to determine what the optimum structure should be.

To aid in any further extensions of this work, the capability to numerically simulate the complex non-linear behaviour of this type of structure has been demonstrated, with the finite element models showing good agreement with the experimental results. To fully demonstrate the feasibility of these structures, further work is required to provide a full empirical demonstration of the probing system's performance. In addition, work is also required to design and implement a control system for active stiffness adjustment. 


\section{Acknowledgments}

The authors wish to acknowledge the funding contribution from the Saudi Arabian cultural bureau in London. Thanks also to Professor Richard Leach (University of Nottingham) for reviewing the manuscript.

\section{References}

[1] Hansen HN, Carneiro K, Haitjema H, De Chiffre L. Dimensional Micro and Nano Metrology. CIRP Ann Manuf Technol. 2006;55(2):721-43.

[2] A Weckenmann and G Peggs and,J.Hoffmann. Probing systems for dimensional microand nano-metrology. Measurement Science and Technology. 2006;17(3):504.

[3] Weckenmann A, Estler T, Peggs G, McMurtry D. Probing Systems in Dimensional Metrology. CIRP Ann Manuf Technol. 2004;53(2):657-84.

[4] Leach R. Fundamental principles of engineering nanometrology. In: Elsevier; 2014. p. Chapter 9.

[5] Bos EJC. Aspects of tactile probing on the micro scale. Precis Eng. 2011 4;35(2):228-40.

[6] Murakami H, Katsuki A, Sajima T, Suematsu T. Study of a vibrating fiber probing system for 3-D micro-structures: performance improvement. Measurement Science and Technology. 2014;25(9):094010.

[7] Meli F, Küng A. AFM investigation on surface damage caused by mechanical probing with small ruby spheres. Measurement Science and Technology. 2007;18(2):496.

[8] Claverley JD, Leach RK. Development of a three-dimensional vibrating tactile probe for miniature CMMs. Precis Eng. 2013;37(2):491-9.

[9] Lewis AJ. Fully traceable miniature CMM with submicrometer uncertainty. Optical Science and Technology, SPIE's 48th Annual Meeting; International Society for Optics and Photonics; 2003.

[10] Fan K, Cheng F, Wang W, Chen Y, Lin J. A scanning contact probe for a microcoordinate measuring machine (CMM). Measurement Science and Technology. 2010;21(5):054002.

[11] Ruther P, Bartholomeyczik J, Trautmann A, Wandt M, Paul O, Dominicus W, et al. Novel 3D piezoresistive silicon force sensor for dimensional metrology of micro components. Sensors, 2005 IEEE; IEEE; 2005.

[12] He M, Liu R, Li Y, Wang H, Lu X, Ding G, et al. Tactile probing system based on micro-fabricated capacitive sensor. Sensors and Actuators A: Physical. 2013;194:128-34. 
[13] Kim K, Liu X, Zhang Y, Sun Y. Nanonewton force-controlled manipulation of biological cells using a monolithic MEMS microgripper with two-axis force feedback. $\mathrm{J}$ Micromech Microengineering. 2008;18(5):055013.

[14] Challa VR, Prasad M, Shi Y, Fisher FT. A vibration energy harvesting device with bidirectional resonance frequency tunability. Smart Mater Struct. 2008;17(1):015035.

[15] Bonello P, Brennan MJ, Elliott SJ, Vincent JF, Jeronimidis G. Designs for an adaptive tuned vibration absorber with variable shape stiffness element. Proceedings of the Royal Society A: Mathematical, Physical and Engineering Science. 2005;461(2064):3955-76.

[16] Leland ES, Wright PK. Resonance tuning of piezoelectric vibration energy scavenging generators using compressive axial preload. Smart Mater Struct. 2006;15(5):1413.

[17] Alblalaihid K, Kinnell P, Ratchev S. An evaluation of stiffness modulating techniques for a micro-CMM tactile probe. Conference Proceedings - 14th International Conference of the European Society for Precision Engineering and Nanotechnology, EUSPEN 2014; ; 2014.

[18] Pril WW. Development of high precision mechanical probes for coordinate measuring machines [dissertation]. Eindhoven: Technische Universiteit Eindhoven; 2002.

[19] Welsch G, Boyer R, Collings E. Materials properties handbook: titanium alloys. ASM international; 1993.

[20] Alblalaihid K, Kirk T, Lawes S, Kinnell P. Fabrication of a Smart Suspension Structure of Micro Tactile Probing. Procedia Engineering. 2014;87:1164-7.

[21] Chu C, Chiu C. Development of a low-cost nanoscale touch trigger probe based on two commercial DVD pick-up heads. Measurement Science and technology. 2007;18(7):1831. 